Article

\title{
Flow Characteristics for Two-Strand Tundish in Continuous Slab Casting Using PIV
}

\author{
Jun Huang *, Zhigang Yuan, Shaoyuan Shi, Baofeng Wang and Chi Liu * \\ School of Energy and Environment, Inner Mongolia University of Science and Technology, Baotou 014010, \\ China; 18940046801@163.com (Z.Y.); shishaoyuan@126.com (S.S.); 13327185758@163.com (B.W.) \\ * Correspondence: hjun8420@imust.edu.cn (J.H.); liuchiyd@163.com (C.L.); \\ Tel.: +86-472-595-1568 (J.H.); +86-131-9070-8932 (C.L.)
}

Received: 4 January 2019; Accepted: 14 February 2019; Published: 17 February 2019

\begin{abstract}
With the development of continuous casting technology, there has been an increase in the stringent requirements for the cleanliness and quality of steel being produced. The flow state of molten steel in tundish is the key to: Optimizing the residence time of molten steel in the tundish; homogenizing the temperature of molten steel; and removing inclusions by floatation. Hence, from theoretical and practical aspects, it is imperative to examine and analyze the flow field of molten steel in the tundish in order to ensure the desired molten steel flow. In this study, a two-strand tundish with $650 \mathrm{~mm} \times 180 \mathrm{~mm}$ slab casting is considered as the subject for this research. According to the similarity theory, combined with the geometrical shape and dimension of the prototype tundish, a tundish model with a geometric similarity ratio of 2:3 is established in the laboratory. Digital particle image velocimetry (PIV) is employed to measure and examine the flow fields at different casting speeds for a tundish containing different flow control devices. The flow in the tundish is typically turbulent and also consists of a vortex motion; it exhibits both random and ordered characteristics. Results reveal that the presence of baffles with $15^{\circ}$ holes can cause an upward-directed flow in the outlet section and give rise to a large circulation. When the casting speed is doubled, the overall velocity of the flow field and turbulent intensity increase, leading to an increase in the molten steel surface velocity.
\end{abstract}

Keywords: steel tundish; baffle; flow field; velocity; PIV

\section{Introduction}

The main function of the tundish is to act as a steel reservoir between the ladle and the mold, and in the case of multistrand casters, to distribute the liquid into the molds. In addition to being a reservoir of liquid steel, the tundish is more increasingly used as a metallurgical reactor vessel aimed at improving control of steel cleanness, temperature, and composition. Tundish metallurgy was proposed in the early 1980s as a special secondary refining technology and an important link in ensuring excellent steel quality during production from smelting and refining to the formation of slabs or billets [1]. In the past three decades, many experts and researchers have done a lot of work and published many important papers. A comprehensive review was given in the paper by Sahai [2] and the book by Sahai and Emi [3].

The basic physical phenomenon in the tundish involves the flow of molten steel. The metallurgical effect of the tundish is mainly achieved by the reasonable flow of molten steel in the tundish. The flow is intended to deliver the molten metal to the molds evenly and at a designed throughput rate and temperature with minimal contamination by, and maximize flotation of macro inclusions. Hence, the investigation of the flow phenomenon in the tundish is the foundation of tundish metallurgy. 
Physical modeling has played a key role in tundish research [4-6]. In physical modeling, a low temperature aqueous analog, generally water, is used to represent molten metal in a tundish. In particular, the kinematic viscosity of water is similar to that of molten steel. Water flow in a transparent model tundish can be used to observe melt flow physically taking place in an actual tundish. A full or reduced scale tundish model may be designed based on appropriate similarity criteria in which the flow of molten metal is simulated by the flow of water. As water flow in the model is a realistic representation of the actual tundish melt flow, it may be used to study the melt flow in a tundish.

According to the similarity principle for flow phenomena, the use of a water model to investigate the flow of molten steel in tundish is not only feasible, but also can accurately reflect the values and law of the actual flow of molten steel. However, in the actual hydraulics simulation of a tundish, it is difficult to completely maintain all forces to be equal between the model and the prototype; hence, different situations need to be considered. In a water simulation experiment using a reduced-scale model, it is imperative to use an approximate model method to ensure that the flow in the model is similar to that in the prototype.

Based on flow visualization and image processing technology, a digital image technique has been developed, which combines single point measurement technology (hotline, turbine) and flow visualization technology. Digital image measurement technology can not only achieve the overall structure and transient image field, but also obtain the velocity data of the whole flow field quantitatively. Some researchers [7-9] have measured the flow field in the tundish by the particle image velocimetry (PIV), thus that the overall structure and transient image of a planar flow field was obtained quantitatively.

Many attempts have been made to improve melt flow characteristics in existing tundish's by the installation of various flow control devices, such as weirs, dams, baffles with holes, and turbulence suppressors. The beneficial effects of various flow modification devices have been applied in actual industrial trials as well as physical and mathematical modeling studies [10,11]. Optimum placement of flow control devices has been found to result in an increase in the average residence time of fluid as well as an increase in the plug flow volume in the tundish. These flow control devices, properly installed, may create localized mixing in contained regions, which may help in inclusion agglomeration and hence, inclusion removal.

Due to the limited of view of the camera in PIV, developing an approach for measuring a water model with a length close to $5 \mathrm{~m}$ is an important problem to be solved in this paper. In this study, the PIV flow measurement is carried out for a reduced-scale two-strand tundish model for slab production. The paper analyzes the flow characteristics in a two-strand tundish for continuous slab casting with different flow-control devices. The effect of eddies is discussed.

\section{Experimental Object and Scheme}

To transfer results of measurements from a model to the original tundish, apart from the geometric similarity, the fluid-dynamic similarity must be considered as well. For an isothermal water model experiment, geometrical similarity, and dynamic similarity between the model and the prototype are required. In order to maintain similarity in depth of liquid, the relationship between the velocities and hence the inlet flow rate of the fluid in the model and in the prototype is to be obtained by following either of Froude ( $F r)$ similarity or Reynolds (Re) similarity. For the dynamic similarity, the Re number and Fr number in the model should be equivalent to those in the prototype. As steel flow in the tundish is gravity-driven, it is understood that flow inside the tundish is Froude criteria dominated. As the flow of liquid steel within the tundish is severely turbulent, the Re number of the model is in the same self-modelling region as the prototype, the Re number can meet the requirement naturally. Thus, most reduced scale-modeling studies are done mainly based on Froude similarity criteria [12].

In the experiments, a two-strand tundish of a stainless-steel slab continuous casting is used as the experimental object. The tundish model in the hydraulics experiment comprised clear glass with a 
$2: 3$ ratio to the prototype. The flow rate passing the long nozzle in the experiment is determined on the basis of the typical sectional dimension of the cast slab, i.e., $180 \mathrm{~mm} \times 650 \mathrm{~mm}$, and casting speed. The typical casting speed is $1.2 \mathrm{~m} / \mathrm{min}$. The long nozzle diameter is $75 \mathrm{~mm}$. The main dimensions of the water model are shown in Figure 1, all dimensions are in millimeters. The scale factor of 2:3 is defined as the ratio of lengths in the model and prototype systems to ensure geometric similarity.

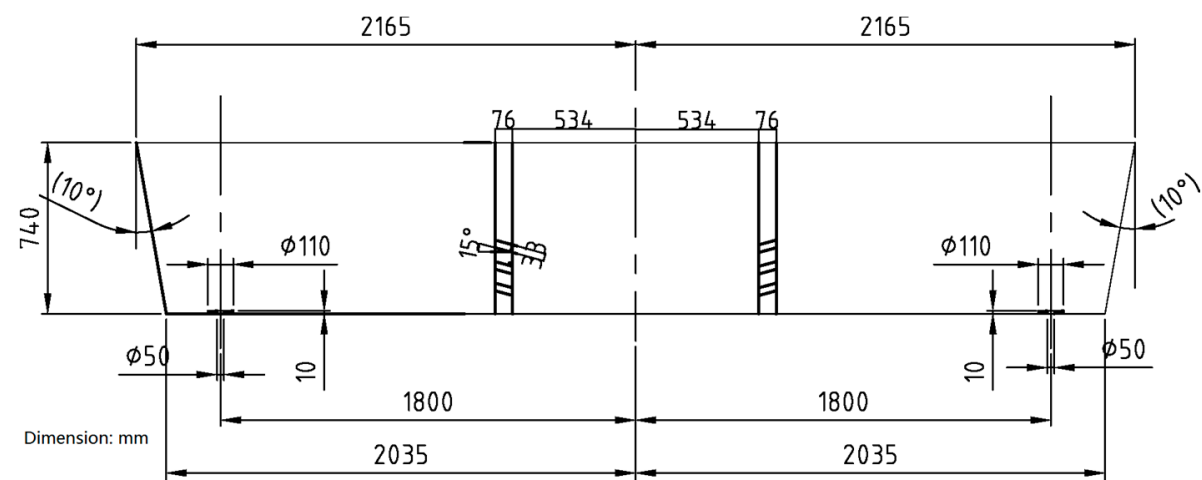

(a)

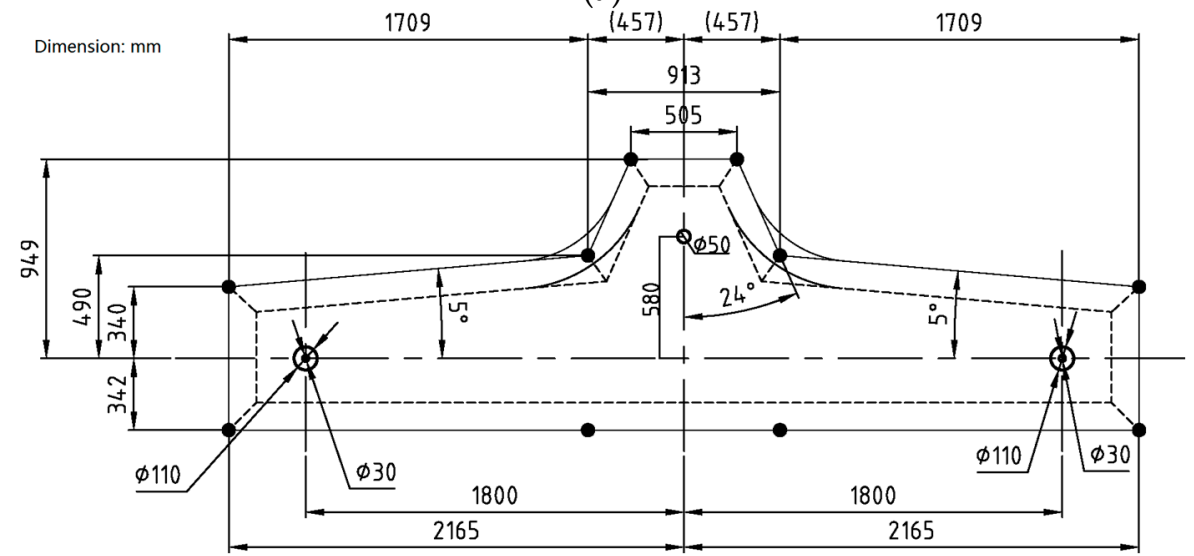

(b)

Figure 1. Two-strand tundish water model (a) shell diagram; (b) control device.

To satisfy the Froude similarity, the Froude number of the water model should be equal to that of the prototype:

$$
F r=\frac{v^{2}}{g l}=\frac{v^{\prime 2}}{g l^{\prime}}
$$

The similarity ratio in velocity can be obtained (model velocity/prototype velocity):

$$
\frac{v^{\prime}}{v}=\sqrt{\frac{l^{\prime}}{l}}=\lambda^{0.5}=\left(\frac{2}{3}\right)^{0.5}
$$

The similarity ratio in the flowrate can be obtained (model flowrate / prototype flowrate):

$$
\frac{Q^{\prime}}{Q}=\frac{l^{\prime 2} \times v^{\prime}}{l^{2} \times v}=\lambda^{2.5}=\left(\frac{2}{3}\right)^{2.5}
$$

where $v$ and $v^{\prime}$ represent the velocity of the fluid in the tundish in the prototype and model respectively, $\mathrm{m} / \mathrm{s} ; g$ is the gravitational acceleration, $\mathrm{m} / \mathrm{s}^{2} ; l$ is the characteristic length, $\mathrm{m} ; \lambda$ is the similarity ratio, $Q$ and $Q^{\prime}$ are the flowrate in the tundish in the prototype and model respectively, $\mathrm{m}^{3} / \mathrm{h}$.

In the water simulation experiment, the quantity of poured steel is controlled by the flowrate at the long nozzle, meanwhile the liquid level is controlled by the level gauge in the tundish model. Table 1 summarizes the concrete experimental parameters of the tundish prototype and the water model. 
Table 1. Experimental parameters of the tundish prototype and water model.

\begin{tabular}{ccccc}
\hline Object & $\begin{array}{c}\text { Melt Depth in } \\
\text { Tundish } / \mathbf{m m}\end{array}$ & Volume $/ \mathbf{m}^{\mathbf{3}}$ & $\begin{array}{c}\text { Long Nozzle Inserted } \\
\text { Depth/mm }\end{array}$ & Flowrate $/\left(\mathbf{m}^{\mathbf{3}} / \mathbf{h}\right)$ \\
\hline Prototype & 900 & 6.00 & 250 & 8.42 \\
Model & 600 & 1.78 & 167 & 3.06 \\
\hline
\end{tabular}

Fluid flow characteristics in the tundish with the incorporation of flow control devices is dependent on the optimum location and size. Numerical simulations have been used to optimize current control devices and installation locations. The optimized results are obtained by numerical simulation and must be validated by actual water simulation experiments. Scheme A is an optimization scheme, while the other three are in contrast to it. The installation location and size of these devices is shown in Figure 1. The flow control devices in the tundish mainly include the baffles with holes and the turbulence suppressor. Table 2 summarizes the experimental scheme. Experiments are carried out in the presence or absence of the flow control device and different casting speeds. The baffles with holes are placed in the middle of the tundish. The baffles are perpendicular to the walls of the tundish. Each baffle has six circular holes with a diameter of $33 \mathrm{~mm}$ and an upward inclination of $15^{\circ}$. To highlight the effect of the flow control device on the flow, the casting speed is doubled for comparing and manifesting the results obtained from the flow test.

Table 2. Experimental scheme and relevant processing parameters of tundish.

\begin{tabular}{ccc}
\hline No. & Flow Control Device & Prototype Casting Speed $/(\mathrm{m} / \mathbf{m i n})$ \\
\hline A & baffles with holes and suppressor & 1.2 \\
B & baffles with holes and suppressor & 2.4 \\
C & Suppressor only & 1.2 \\
D & Suppressor only & 2.4 \\
\hline
\end{tabular}

The model tundish is placed on the experimental platform, and water pipelines are connected. After setting the experimental parameters and waiting for $30 \mathrm{~min}$ for the flow to become steady, PIV flow measurement starts.

According to the flow path of the molten steel in the tundish, the measuring section is shown in Figure 2. The measurement section is sequentially scanned with the PIV. The photographs by PIV are processed by PIVview software, then the flow field diagrams from different locations are merged into the full section. PIVview is a compact program package for the evaluation of particle image velocimetry with the PIV-Groups of the German Aerospace Center. The software is be developed in close cooperation with the Institute of Aerodynamics and Flow Technology of the German Aerospace Center in Goettingen, Germany.

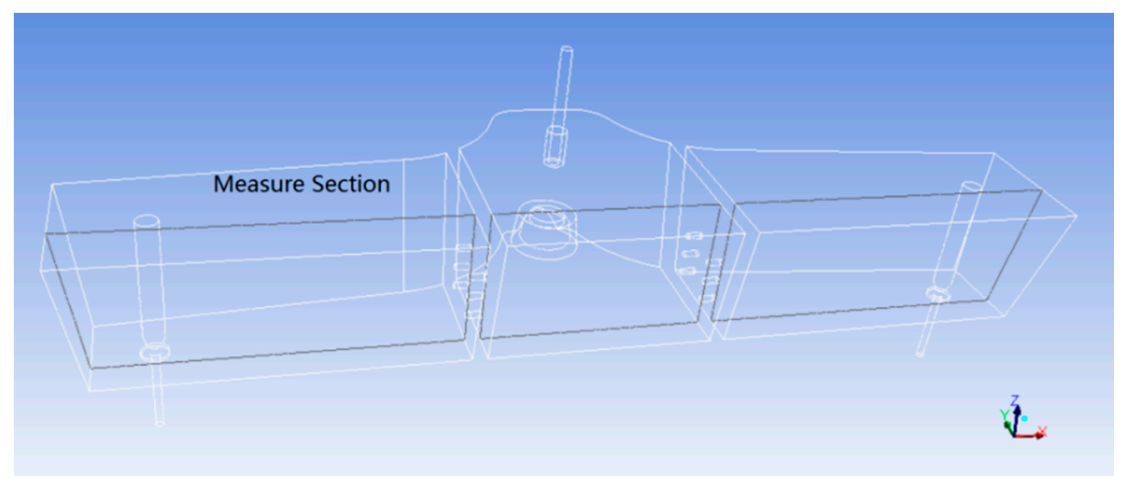

Figure 2. Schematic of the measuring section positions in the tundish. 


\section{PIV Measurement Process}

Figure 3 shows the hardware structure diagram of the test system developed in this study for the flow field. A certain number of tracing particles are evenly dispersed in the flow field. The 2D flow field with an approximate thickness of $3 \mathrm{~mm}$ is illuminated by a sheet light source comprising a laser and its lens. Two high-resolution cameras are arranged along the vertical direction of the laser to form a camera array, which can record the trajectory of the tracing particles of a particular frequency under the illumination of the sheet light source. The time interval between the two pulses of the laser that illuminate the flow field is set to meet the camera's recording frequency and synchronize the camera's recording frequency with the laser's stroboscopic frequency using the synchronizer, which are similar to commercial PIV requirements in that two continuous frames can be continuously recorded in a short time period. The use of a multi-camera array can meet the measurement for a large sight field. The machine vision systems are employed related to the acquisition and processing of data during the image recognition and velocity measurements, enabling the system to solve low-speed measurement problems at a lower cost. The motion drive unit controlled by the servo motor can meet the requirements of segmentation measurement. Combined with the camera array setting, it can meet the measurement requirement for velocity in a wider range, which also provides the necessary hardware and software conditions for implementing the different scale measurement for the flow field in the tundish.

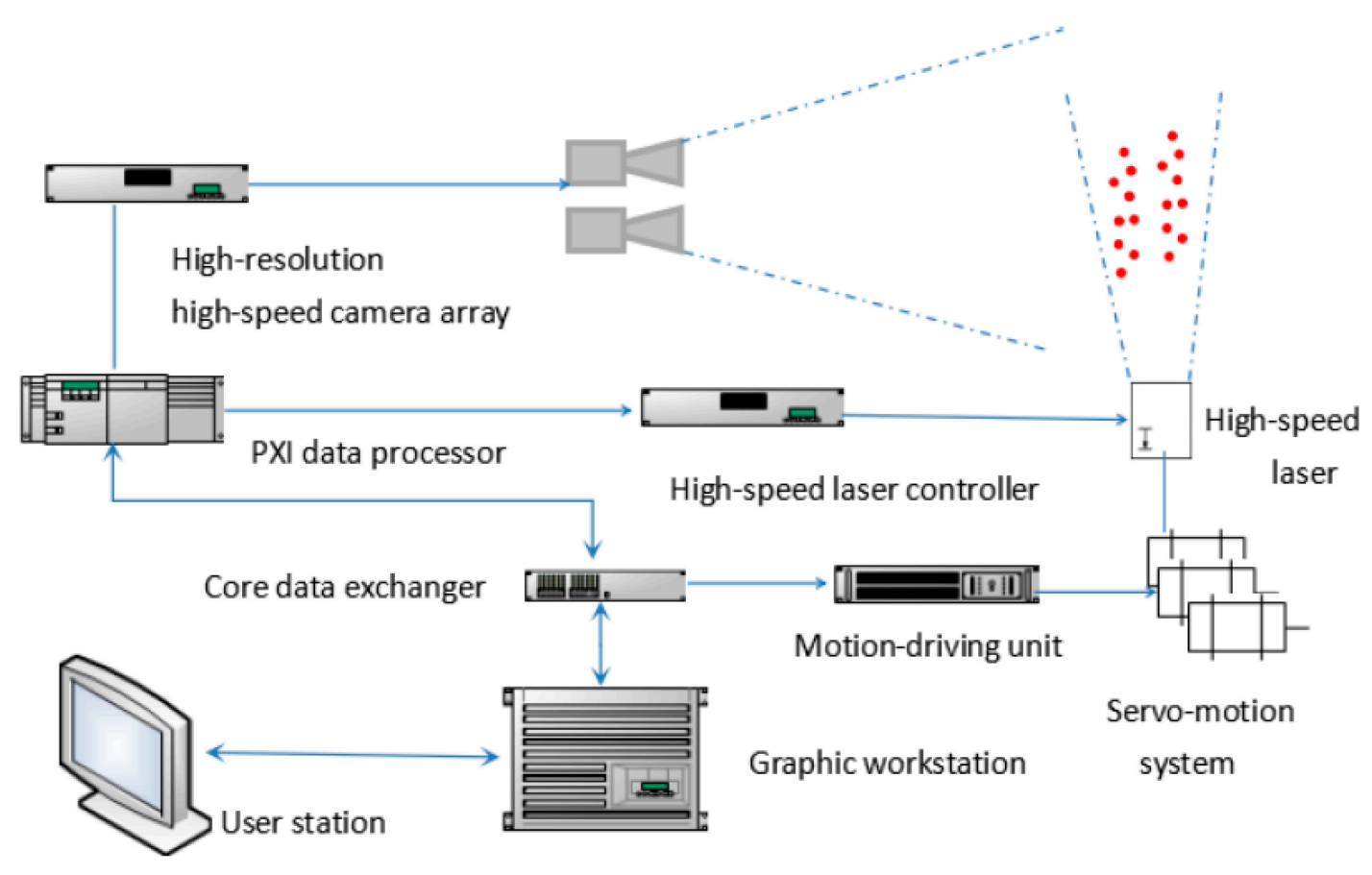

Figure 3. Diagram of the particle image velocimetry (PIV) system.

Owing to the low flow velocity in the tundish (average velocity $<1.0 \mathrm{~m} \cdot \mathrm{s}^{-1}$ ), the continuous shooting mode of a digital camera with a fixed number of frames can meet the requirement to trace particles. The method does not require two consecutive frames in an extremely short time (5 $\mu$ s) as it does by the PIV technology and only needs to ensure the synchronization between the continuous shooting speed of the digital camera and the laser strobeflash, hence, the measurement system can not only decrease the price, but also the array comprising multiple cameras can meet the requirements for obtaining a large field of view simultaneously. The image acquisition system for the flow field in the experimental tundish mainly comprises of an MGL-N-532 laser source, two Canon 5D MARK III cameras (Tokyo, Japan) and the background control and data processing software, which is shown in Figure 4. The laser emitted by the MGL-N-532 laser is the green laser with a wavelength of $532 \mathrm{~nm}$ 
and a shooting frequency of $30 \mathrm{~Hz}$. Polystyrene beads with a density of $1.02 \mathrm{~g} / \mathrm{cm}^{3}$ and a diameter of $50 \mu \mathrm{m}$ are tracing particles. Similar to a commercial PIV system, the displacement of tracing particles is obtained by the cross-correlation algorithm for the two images in the tested area, and the data are processed by the authorized PIVview software to display the two-dimensional velocity vector field within the areas in which velocity is measured [13].

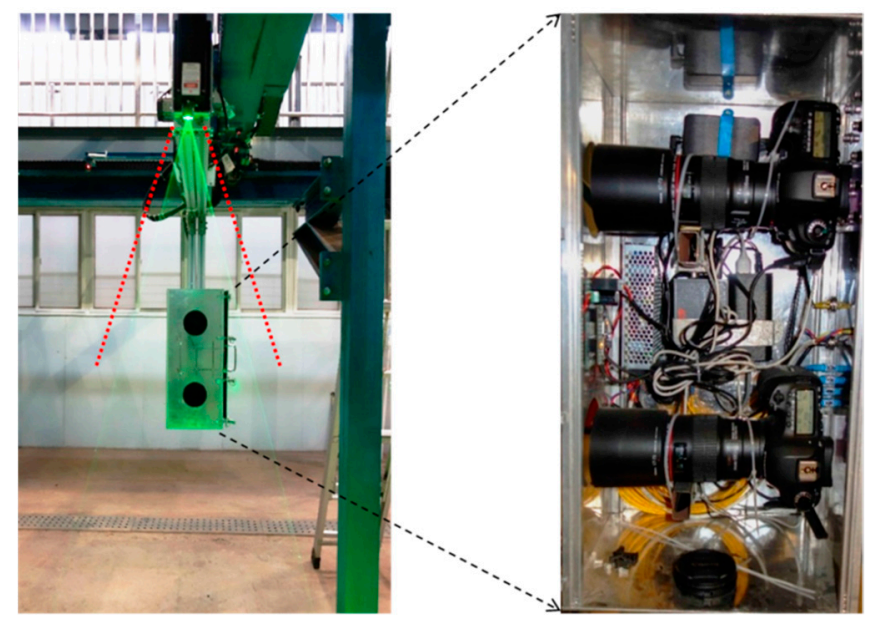

Figure 4. The image acquisition system.

As the sectional flow field of the tundish water model exceeds the shooting range of the camera array, the sectional flow field of the entire tundish water model must be obtained by the image stitching method. Although the measuring moments for different areas are not the same, the stitched image can reflect the flow distribution characteristics in the cross section.

\section{Experimental Results and Discussion}

Figure 5 shows the sectional flow field of the tundish with PIV measurements and numerical simulation under schemes A. The position of the measured and simulated results is the same. The simulation shows only half. The position of two baffles with holes is indicated in the diagram. Of the black stripes that appear in the PIV measurement drawing, three are the supporting frames in the process of making the tundish model. The white stripes represent the results of different cameras at different times in Figure 5c. The results obtained from the different method are shown together.

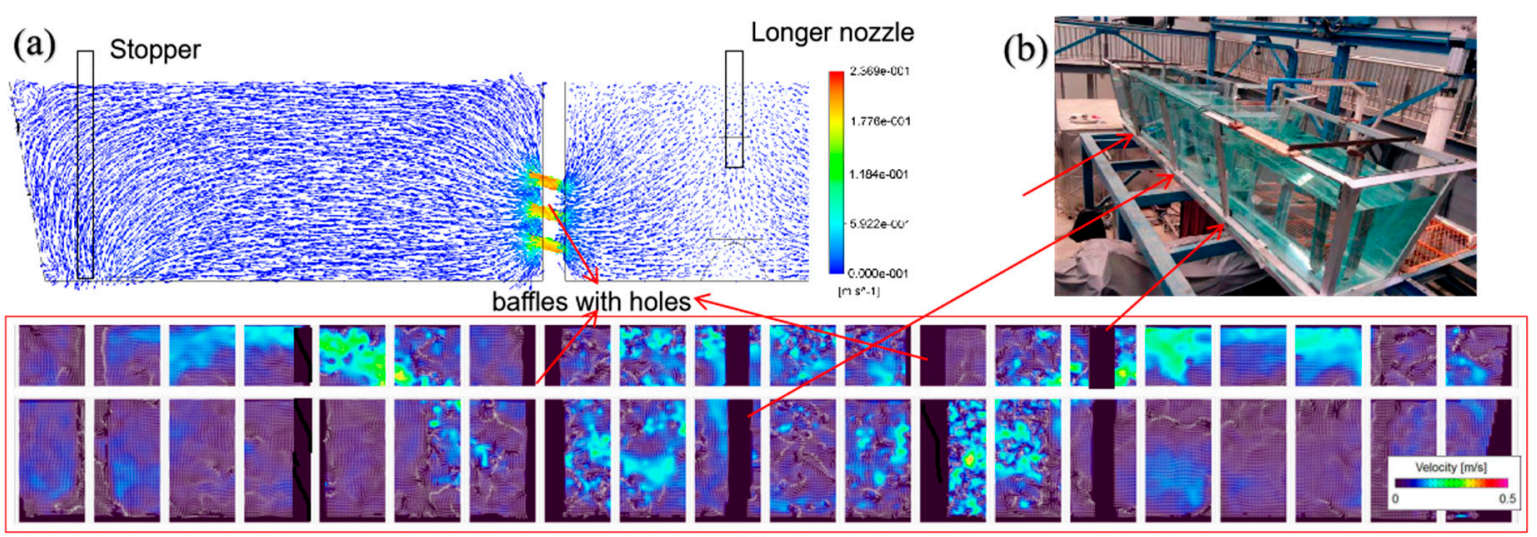

(c)

Figure 5. Flow measurement and simulation result of scheme A; (a) numerical simulation; (b) experimental setup; (c) PIV measurements. 
Figure $5 \mathrm{c}$ shows a very detailed velocity field in which the flow is more active in the inlet section. The very marked large circulation is readily apparent from the plot. It can be seen that flow in the tundish is a local pulse, partially rendering a lot of mixing, but the overall flow shows the trend of a certain flow movement.

Figure 5 a also shows the flow field for the numerical simulation results obtained by test scheme A. The commercial CFD package FLUENT with ANSYS 15.0 is used to model and solve the tundish fluid flow and it is compared with the available experiment results. The tundish with a long nozzle and exit is modelled. A non-uniform Cartesian mesh is used, with a power-law expansion of cells from the walls outwards. The finest cells are placed next to the jet-impact wall, since that is where maximum resolution is needed for the fast-moving boundary layers. A fine mesh is also used to cover the jet region. In order to test the grid independence of the results, it is necessary to test the sensitivity of the results on the number of mesh points used. A total of about 8,500,000 cells are used in the finest calculation. The mathematical model is based on the assumptions of continuum hypothesis, the standard two equation model, $k-\varepsilon$ equation is used to model the turbulence. A steady-state incompressible solution is adopted, with the main dependent variables being the pressure, three velocity components and the two turbulent quantities. The SIMPLE (Semi-Implicit Method for Pressure Linked Equations) algorithm is used for the pressure-velocity coupling and QUICK (Quadratic Upstream Interpolation for Convective Kinetics) scheme is used for discretization of momentum, turbulent equations.

As can be observed from the measurement results, the flow complexity in the tundish is far from the simple recirculating flow that is obtained by the Reynolds-averaged Navier-Stokes (RANS) equations, plus an appropriate turbulence model, combining large circulation and small-scale eddies. To gain more knowledge about the transient turbulence process, which cannot be achieved via Reynolds-averaged equations solutions, large-eddy simulations (LES) of the tundish flow field are performed. Alkishriwi et al. $[14,15]$ and Jha et al. $[16,17]$ have carried out such simulations to investigate the turbulent flow structure and vortex dynamics. They have confirmed that the metallurgical effect of the tundish is mainly accomplished by the flow behavior. LES simulation of the flow field in a tundish is conducted to analyze the flow structure, which determines to a certain extent the steel quality. Many intricate flow details have not been observed by customary RANS approaches.

The motion of the liquid steel is generated by jets into the tundish and continuous casting mold. The flow regime is mostly turbulent, but some turbulence attenuation can occur far from the inlet. The characteristics of the flow in a tundish include jet spreading, jet impingement on the wall, wall jets, and an important decrease of turbulence intensity in the core region of the tundish far from the jet. Compared to the numerical simulation, physical simulation for the flow field provides more details on the evolution of not only velocity but also eddies. Mathematical modeling may provide a much more detailed picture of velocity, turbulence, and temperature fields as a function of location and time. In general, the numerical simulation shows the flow trend, while the water simulation work presents flow details. The two means complement each other and provide a comprehensive understanding of the flow.

Figures 6-8 shows the flow for schemes B, C, and D. C and D do not have baffles with holes. These results also show turbulent flow behavior in the tundish. Eddies can be clearly seen by measurement. Moreover, the evolution and interaction between eddies can be observed by the measurement of a large amount of transient data.

As can be seen from the results of the flow field in the tundish, the flow is typically turbulent and consists of eddies in motion. Turbulence exhibits random and orderly characteristics. One of its basic structures includes the eddies having various scales. Statistically, a large number of random small eddies form the background flow field, and large-scale eddies structures with quasi-ordered structures are statistically significant. The large eddies are limited and affected by flow boundary conditions and flow interfaces. The large eddies contain the turbulent kinetic energy, which is associated with the fluctuating velocity components. The small eddies, which are called the micro scale of turbulence, sometimes do not show up due to the limitation of image sampling setting and image resolution in 
PIVview. According to turbulence theory, the turbulent kinetic energy is being dissipated in the smaller eddies. The small eddies may also play an important role in promoting the coalescence of inclusion particles [12].

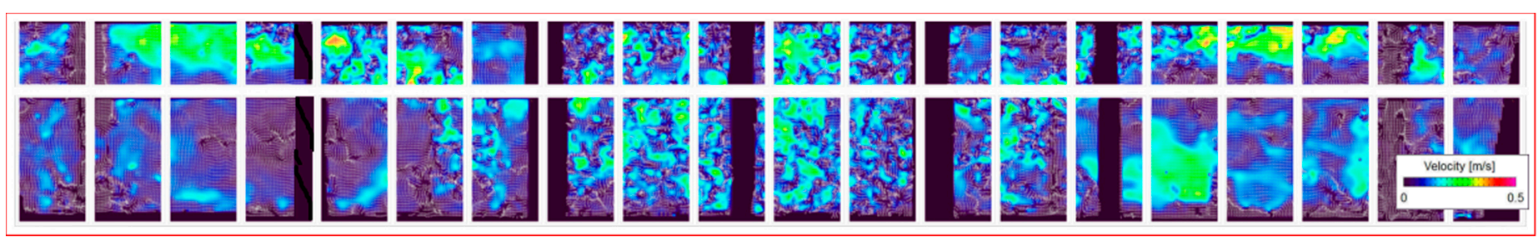

Figure 6. Flow measurement results obtained by scheme B.

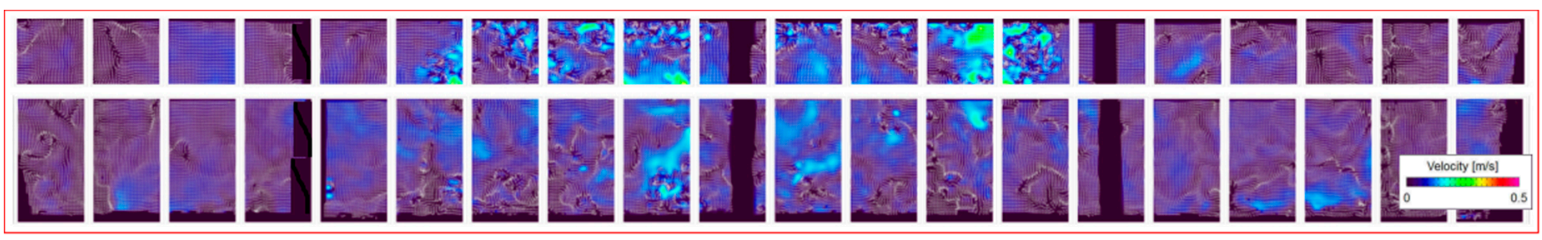

Figure 7. Flow measurement results obtained by scheme C.

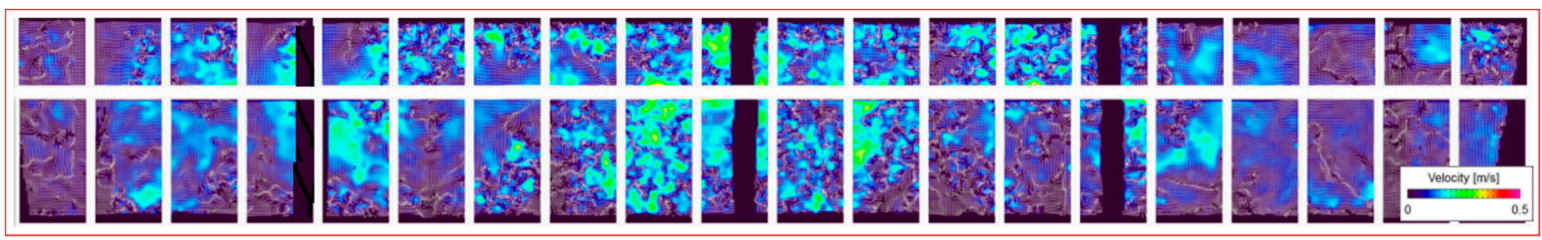

Figure 8. Flow measurement results obtained by scheme D.

The very important manifestation of turbulent flow is the presence of the eddies. The turbulent fluctuations will bring about a very effective mixing, mass (and also heat and momentum) can be readily transported by the eddies from one part of the fluid to the other [12]. The metallurgical effect of the tundish needs to be exerted via the transmission effect of these eddies.

The eddy is a flowing form, the vorticity is a physical quantity. The mathematical definition of vorticity is extremely clear. The curl of the flow field is defined as the vorticity:

$$
\vec{\omega}=\nabla \times \vec{V}
$$

where $\vec{V}$ is the velocity, $\mathrm{m} / \mathrm{s} ; \vec{\omega}$ is the vorticity, $1 / \mathrm{s} ; \nabla$ is the nabla operator. From the definition of vorticity, vorticity is caused by the velocity gradient in the flow field. The positions at which the velocity gradients are large exhibit high strain rates according to Newton's law of viscosity, the flow's viscosity depends on the strain rate. A causal relationship clearly exists between the vorticity and viscosity of the fluid. For homogeneous incompressible fluids, the vorticity is generated from the fluid-solid interface. Besides moving with the flow, the vorticity diffuses like heat. At the inlet section of the tundish, the impacting jet on the turbulence suppressor generates a large number of eddies. Owing to the fluid viscosity and flow control device, a large number of eddies are generated. For preventing the entrapped slag, the molten steel is required to flow out of the tundish outlet in a stable manner. With respect to the aspects of satisfying the requirements of process and the improvement on the metallurgical effect, the application of either a flow control device or a plug rod or increase in the distance between the inlet and outlet can avoid the interference of the inlet's strong vortex flow on the outlet.

For a casting speed of $1.2 \mathrm{~m} \cdot \mathrm{s}^{-1}$ the theoretical velocity in the outlet section can be calculated to be $0.001 \mathrm{~m} \cdot \mathrm{s}^{-1}$ according to the flow rate and sectional area of the tundish. At such low relative average velocity, it is difficult to produce relatively intense heat, mass, and momentum transfer. However, 
owing to the strong impacting jet action occurring near the long nozzle and the effect of the flow control device, the turbulence occurring in the tundish is clear, and a large number of eddies exist.

By the comparison between the presence and absence of the baffles with holes, the flow control device exerts a fundamental change in the flow within the tundish. In the absence of the flow control device, the turbulent areas concentrate near the long nozzle. According to the analysis related to the reason for the generation of the flow eddies in the tundish, owing to the presence of the flow control device inside the tundish and the number of fluid-solid interfaces increasing, the vorticity generating positions increase, intensifying the momentum exchange of the internal flow and making the overall flow easily uniform.

In the presence of the baffles with holes, the flow is in accordance with the requirement of the designed flow control device, especially in the flow direction and flow velocity. After flow passes through the inlet section, the flow is "thrown up" through the $15^{\circ}$ holes to the outlet section surface and flows out from the outlet after sufficient exchange with the surface. Simultaneously, a large circulation is formed in the outlet section. The flow control devices, properly installed, may create localized mixing in contained regions, which may help in inclusion agglomeration and hence, their removal [18].

By doubling the casting speed, the shape of the flow does not significantly change. The overall velocity of the flow field and the turbulent intensity increase. In the presence of the flow control device, the surface velocity in water simulation can reach $0.3 \mathrm{~m} \cdot \mathrm{s}^{-1}$. After similarity conversion, the velocity of molten steel reaches $0.37 \mathrm{~m} \cdot \mathrm{s}^{-1}$. Such high speed may destroy the slag layer in the industrial production. Higher surface velocity could cause tundish slag entrainment at the slag/metal boundary due to turbulence arising and also cause refractory erosion near the nozzle or stopper, which requires the use of more expensive refractory practices.

By the millimeter-level spatial resolution of the tundish flow and the measurement and display at the millisecond-level time dimension scale, it is possible to achieve the precise understanding of the flow in the tundish. After combining the reality of the eddy structure and the evolution of eddies, an extremely comprehensive and precise understanding on the flow within the tundish can be achieved. These results provide a good precondition for examining the metallurgical effects of other tundishes on the basis of understanding the flow.

\section{Conclusions}

(1) Based on camera array scanning, reduced-scale two-strand water tundish flow fields are obtained at different casting speeds using different flow control devices. Flow measurement results show richer flow-field details than RANS simulation.

(2) There is a typical turbulent flow as well as vortex motions simultaneously in the tundish. The flow-control device and the boundary have an effect on the generation and dissipation of eddies, which has an important influence on the metallurgical effect of the tundish.

(3) Baffles with $15^{\circ}$ holes can cause an upward-directed flow in the outlet section and form a large circulation. Eddies are generated and the flow in the tundish tends to be more uniform. As the casting speed is doubled, the overall velocity of the flow field and the turbulent intensity increase, resulting in a molten steel surface velocity of up to $0.37 \mathrm{~m} / \mathrm{s}$.

Author Contributions: Conceptualization, J.H. and B.W.; methodology, J.H.; formal analysis, Z.Y.; investigation, S.S. and J.H.; resources, J.H.; data curation, S.S. and C.L.; writing-original draft preparation, B.W.; writing-review and editing, J.H.; visualization, C.L. and Z.Y.; supervision, Z.Y.; project administration, J.H.; funding acquisition, J.H. and B.W.

Funding: The work was supported by the China National Heavy Machinery Research Institute Co., Ltd. and Natural Science Foundation of Inner Mongolia under Grant 2017MSLH0534.

Acknowledgments: The author would like to thanks Baotou Lianfang High Tech Co., Ltd. for their technical support.

Conflicts of Interest: The authors declare no conflict of interest. 


\section{References}

1. Mazumdar, D. Tundish metallurgy: Towards increased productivity and clean steel. Trans. Indian Inst. Met. 2013, 66, 597-610. [CrossRef]

2. Sahai, Y. Tundish technology for casting clean steel: A review. Metall. Mater. Trans. B-Process Metall. Mater. Process. Sci. 2016, 47, 2095-2106. [CrossRef]

3. Sahai, Y.; Emi, T. Tundish Technology for Clean Steel Production; World Scientific: Hackensack, NJ, USA, 2008; pp. 1-14.

4. Ramos-Banderas, A.; Sanchez-Perez, R.; Morales, R.D.; Palafox-Ramos, J.; Demedices-Garcia, L.; Diaz-Cruz, M. Mathematical simulation and physical modeling of unsteady fluid flows in a water model of a slab mold. Metall. Mater. Trans. B-Process Metall. Mater. Process. Sci. 2004, 35, 449-460. [CrossRef]

5. Sahai, Y. Advances in tundish technology for quality improvements of cast steel. J. Iron Steel Res. Int. 2008, 15, 643-652.

6. Braun, A.; Warzecha, M.; Pfeifer, H. Numerical and physical modeling of steel flow in a two-strand tundish for different casting conditions. Metall. Mater. Trans. B-Process Metall. Mater. Process. Sci. 2010, 41, 549-559. [CrossRef]

7. Odenthal, H.J.; Bolling, R.; Pfeifer, H. Numerical and physical simulation of tundish fluid flow phenomena. Steel Res. Int. 2003, 74, 44-55. [CrossRef]

8. Giurgea, C.; Bode, F.; Nascutiu, L.; Dudescu, C. Considerations regarding the optically transparent rigid model for piv investigations. A case study. Part 2: Notes on the failure of the model. Energy Procedia 2016, 85, 235-243. [CrossRef]

9. Cwudziński, A. Piv method and numerical computation for prediction of liquid steel flow structure in tundish. Arch. Metall. Mater. 2015, 60,11-17. [CrossRef]

10. Merder, T. Influence of design parameters of tundish and technological parameters of steel continuous casting on the hydrodynamics of the liquid steel flow. Metalurgija 2014, 53, 443-446.

11. Cwudzinski, A. Numerical and physical modeling of liquid steel active flow in tundish with subflux turbulence controller and dam. Steel Res. Int. 2014, 85, 902-917. [CrossRef]

12. Szekely, J.; Ilegbusi, O.J. The Physical and Mathematical Modeling of Tundish Operations; Springer: New York, NY, USA, 1989; pp. 1-52.

13. Huang, J.; Zhang, Y.; Zhang, Y.; Zhang, Y.; Ye, X.; Wang, B. Study of flow characteristics of tundish based on digital image velocimetry technique. Metall. Mater. Trans. B 2016, 47, 3144-3157. [CrossRef]

14. Alkishriwi, N.; Meinke, M.; Schroder, W. A large-eddy simulation method for low Mach number flows using preconditioning and multigrid. Comput. Fluids 2006, 35, 1126-1136. [CrossRef]

15. Alkishriwi, N.; Meinke, M.; Schroder, W.; Braun, A.; Pfeifer, H. Large-eddy simulations and particle-image velocimetry measurements of tundish flow. Steel Res. Int. 2006, 77, 565-575. [CrossRef]

16. Jha, P.K.; Ranjan, R.; Mondal, S.S.; Dash, S.K. Mixing in a tundish and a choice of turbulence model for its prediction. Int. J. Numer. Methods Heat Fluid Flow 2003, 13, 964-996. [CrossRef]

17. Jha, P.K.; Rao, P.S.; Dewan, A. Effect of height and position of dams on inclusion removal in a six strand tundish. ISIJ Int. 2008, 48, 154-160. [CrossRef]

18. Warzecha, M.; Merder, T.; Warzecha, P. Investigation of the flow structure in the tundish with the use of RANS and LES methods. Arch. Metall. Mater. 2015, 60, 215-220. [CrossRef]

(C) 2019 by the authors. Licensee MDPI, Basel, Switzerland. This article is an open access article distributed under the terms and conditions of the Creative Commons Attribution (CC BY) license (http://creativecommons.org/licenses/by/4.0/). 